PACS 32.80.Rm; 05.45.+b;

УДК 539.184, 539.186

\title{
SENSING THE STOCHASTIC LASER PULSE STRUCTURE AND CHAOTIC AND PHOTON-CORRELATION EFFECTS IN THE NON- LINEAR MULTI-PHOTON ATOMIC DYNAMICS IN LASER AND DC ELECTRIC FIELD
}

\author{
A. V. Glushkov, G. P. Prepelitsa and A. A. Svinarenko \\ Institute of Applied mathematics OSEU, P.O.Box 108, Odessa-9, 65009, Ukraine \\ Phone: +380-482-637227 E-mail: glushkov@paco.net
}

\begin{abstract}
SENSING THE STOCHASTIC LASER PULSE STRUCTURE AND CHAOTIC AND PHOTON-CORRELATION EFFECTS IN THE NON-LINEAR MULTI-PHOTON ATOMIC DYNAMICS IN LASER AND DC ELECTRIC FIELD

\section{A. V. Glushkov, G. P. Prepelitsa and A. A. Svinarenko}

A new method for sensing a structure of the stochastic, multi-mode laser pulse field and photon-correlation, chaotic features of atomic and nano-optical systems in this field is developed and a dynamics of multi-photon ionization in intense field is studied. As a basis for developing a new method for sensing stochasticity and chaotic features we use new quantum approach to calculation of the multi-photon resonances spectra characteristics for atomic systems in a stochastic laser field and the same field plus DC electric field. Some numerical illustrations regarding the multi-photon resonance spectra and stochasticity sensing for a number of atomic systems are presented.
\end{abstract}

Key words: laser pulse structure, stochasticity, sensing, atom, stochastic laser and electric field

\section{Анотація \\ ДЕТЕКТУВАННЯ СТРУКТУРИ СТОХАСТИЧНОГО ЛАЗЕРНОГО ІМПУЛЬСУ І ХАОТИЧНИХ, ФОТОН-КОРЕЛЯЦІЙНИХ ЕФЕКТІВ В НЕЛІНІЙНІЙ БАГАТОФОТОННІЙ АТОМНІЙ ДИНАМИЦІ У ЛАЗЕРНОМУ Й СТАЛОМУ ЕЛЕКТРИЧНОМУ ПОЛЯХ}

\section{О. В. Глуиков, Г. П. Препелица, А. А. Свинаренко}

Розвинуто новий метод детекування структури стохастичних багатоомодових лазерних імпульсів і хаотичних, фотон-кореляційних ефектів для атомних і нано-оптичних систем в полі лазерного імпульсу. Вивчається динаміка багатофотонної іонізації в інтенсивних полях. В якості теоретичної основи нового методу детектування використано новий квантовий підхід до обчислення характеристик спектра багатофотонних резонансів для атомних систем у стохастичному лазерному полі й сталому електричному полі. Для ряда атомних систем наведені чисельні приклади обчислення спектру, характеристик багатофотонних резонансів і детектування стохастичності у системі.

Ключові слова: структура лазерного імпульсу, стохастичність, детектування, атомна система, стохастичне лазерне і електричне поле 


\title{
Аннотация \\ ДЕТЕКТИРОВАНИЕ СТРУКТУРЫ СТОХАСТИЧЕСКОГО ЛАЗЕРНОГО ИМПУЛЬСА И ХАОТИЧЕСКИХ, ФОТОН-КОРРЕЛЯЦИОННЫХ ЭФФЕКТОВ В НЕЛИНЕЙНОЙ МНОГОФОТОННОЙ АТОМНОЙ ДИНАМИКЕ В ЛАЗЕРНОМ И ПОСТОЯННОМ ЭЛЕКТРИЧЕСКОМ ПОЛЯХ
}

\author{
А. В. Глуиков, Г. П. Препелица, А. А. Свинаренко
}

\begin{abstract}
Развит новый метод детектирования структуры стохастических многомодовых лазерных импульсов и хаотических, фотон-корреляционных эффектов для атомных и нано-оптических систем в поле лазерного импульса. Изучается динамика многофотонной ионизации в интенсивных полях. В качестве теоретической основы нового метода детектирования используется новый квантовый подход к вычислению характеристик спектра многофотонных резонансов для атомных систем в стохастическом лазерном поле и постоянном электрическом поле. Для ряда атомных систем приведены численные примеры вычисления спектра, характеристик многофотонных резонансов и детектирования стохастичности в системе.
\end{abstract}

Ключевые слова: структура лазерного импульса, стохастичность, детектирование, атомная система, стохастическое лазерное и электрическое поле

\section{Introduction}

In last years a phenomenon of dynamical chaos attracts a great interest as a manifestation of this effect in photo-optical systems may in a significant degree change a functional regime (c.f.[1-20]). Cited effect is usually observed in the physical systems and related to a type of non-linear effects. As a rule, dynamical chaos is manifested in the quantum systems, which are not linear in a classic limit. Above especially effective manifestations of this effect in the quantum systems one could mention systems which interact with external, time dependent, for example laser, field. The dynamical chaos features are discovered near boundary of the discrete spectra and continuum [1-3].

It has been discovered that dynamics of atomic and molecular, cluster and nano-optical systems in a laser field has features of the random, stochastic kind and its realization does not require the specific conditions. The importance of studying a phenomenon of stochasticity or quantum chaos in dynamical systems in laser field is provided by a whole number of technical applications, including a necessity of understanding chaotic features in a work of different electronic devices and systems. An actual problem is sensing the structure of the non-coherent multimode laser pulse field. Another important topic is a problem of governing and control of non-linear processes in a stochastic, multi-mode laser field [3$6]$. The principal aim of quantum coherent control is to steer a quantum system towards a desired final state through interaction with light while simultaneously inhibiting paths leading to undesirable outcomes. This type of quantum interference is inherent in non-linear multi photon processes. Controlling mechanisms have been proposed and demonstrated for atomic and solid-state systems. Recently experimental work of Dudovich etal tests the effect of pulse-shaping on transient populations of the excited $\mathrm{Rb}$ atoms. Gibson performs calculations for threelevel systems and 1D model of a two-electron molecule [4-6]. Transitions to excited state occur via a 12-photon interaction for an $800 \mathrm{~nm}$ intense pulse of length 244 au, or just over 2 cycles. Theoretical studies of the laser-atom non-linear interactions are usually based on solving the time-dependent Schrödinger equation or using the time-independent Floquet formalism. In [6] authors extended the nonHermitian multi-state Floquet dynamics approach of Day et al to treat one-electron atomic system to the case of general multi-electron ones. The result is a generalization of the R-matrix Floquet theory, developed by Burke et al, that allows for pulse shape effects whilst retaining the ab initio treatment of detailed electron correlation. At the same time a problem of correct description of the non-linear atomic dynamics in a stochastic, multi-mode laser field is quite far from the final solution. It requires developing principally new approaches to description of multi-photon dynamics and new scheme for sensing the stochasticity and photon-correlation effects. 
Here we develop a new method for sensing a structure of the stochastic, multi-mode laser pulse and chaotic, photon-correlation effects in the atomic, nano-optical systems in a laser and also laser and DC electric field. The results of studying multi-photon ionization dynamics in external field are presented. As a basis for developing a new method for sensing stochasticity and photon-correlation features we use new quantum approach to calculating the multi-photon resonances spectra characteristics for atomic systems in a stochastic laser field and also DC electric and laser field.

\section{Structure of the multi-mode laser pulse. S-matrix energy approach to atomic system in the stochastic multi-mode laser field and DC electric filed}

As it is well known, for a laser with more than one longitudinal mode, mode beating gives rise to intensity fluctuations within the laser pulse $[9,11]$. The beat frequencies for $\mathrm{n}$ modes range up to $n c / 2 L=B$, where $\mathrm{L}$ is the optical length of the laser oscillator. A detailed analysis of the mode structure of the typical dye laser [9] shows that it has about 15 modes, separated by $1 \mathrm{GHz}$ with a Gaussian amplitude distribution. Classically, the field can be written as follows:

$$
E(t)=\varepsilon(t) e^{-i \omega t}+\text { c.c. },
$$

where

$$
\varepsilon(t)=\sum_{i} 0.5 a_{i}(t) e^{-i\left(\Delta \omega_{i} t+\phi_{i}\right)}
$$

Each mode has amplitude $a_{i}$ containing a gaussian time envelope, a frequency detuning $\Delta \omega_{i}$ from the central laser frequency and phase $\phi_{i}$. As experimental study $[9,11]$ of described laser pulse showed that there is no evidence of phase coherence in the temporal behaviour of the laser pulse and thus it is usually assumed that the modes have random phases. Further to make sensing a stochastic structure of the multi-mode laser pulse it is possible to consider an interaction "atomic system - stochastic multimode laser pulse" (or system "an atom-laser pulseDC electric field"). Below it will be shown that this interaction is characterized by availability of the specific chaotic, photon-correlation effects. New theoretical scheme for sensing stochasticity and photon-correlation features is based on the S-matrix energy approach [13-18] to calculating the multiphoton resonances spectra characteristics for atomic systems in a stochastic laser field and also DC electric and laser field. Note that the last case is at first considered in this paper.

Let us present the corresponding theoretical scheme. Following to ref. [13-18], we describe the interaction of atom with the realistic laser field not by means the separated atomic levels shifts and by another set of characteristics, which are directly observed in the experiment. We are studying the radiation emission and absorption lines. Its position and shape fully determine the spectroscopy of atom in the field. It is natural to describe these lines by there moments of different orders $\mu_{n}$. The moments $\mu_{n}$ are strongly dependent upon the laser pulse quality: intensity and the mode constitution. In particular, the k-photon absorption line center shift in the transition $\alpha \rightarrow p$ can not be obtained from the corresponding expressions for the «one»-photon absorption by the change $\omega_{0} \rightarrow \omega_{0} / k$ and introduction of the multiplier $1 / \mathrm{k}\left(\omega_{0}-\right.$ the central laser emission frequency). The difference arises already in the first non-appearing perturbation theory (PT) order and connects with the unusual behaviour of the dynamic polarizability of atom in the resonant range $[15,16]$.

Let us describe the interaction of atom with laser radiation by means the following potential:

$$
V(r, t)=V(r) \int d \omega f\left(\omega-\omega_{0}\right) \sum_{n=-\infty}^{\infty} \cos \left[\omega_{0} t+\omega_{0} n \tau\right],
$$

where $n$ is the whole number. The potential $V$ represents the infinite duration of laser pulses with known frequency $\tau$. Here we consider the effects of interaction of the atom with the single pulse.. The representation $V(r t)$ as the infinite sequence of pulses is a formal moment connected with the application of the stationary PT formalism. The function $f(\omega)$ is a Fourier component of the laser pulse. The condition $\int d \omega f^{2}(\omega)=1$ normalizes potential $V(r t)$ on the definite energy in a laser pulse. Let us consider the pulse with Lorentzian shape (coherent 1-mode pulse): $f(\omega)=N /\left(\omega^{2}+\Delta^{2}\right)$, Gaussian shape (multi-mode chaotic laser pulse): $f(\omega)=N \exp \left[\ln 2\left(\omega^{2} / \Delta^{2}\right)\right]$, and soliton-like pulse of the following shape: $f(\mathrm{t})=$ $=N \mathrm{ch}^{-1}[t / D]$. Besides, in further we will apply the DC electric field simultaneously with laser pulse. An availability of the electric field allows to strengthen the stochastic features in system. The further program resulted in the calculating for any atomic level imaginary part of energy shift Im $E_{\alpha}\left(\omega_{0}\right)$ as the function of the laser pulse central frequency. An according function has the shape of the resonant curve. Each resonance is connected with 
the transition $\alpha-p$, in which the definite number of photons are absorbed or radiated. Let us consider following situation: $\alpha-p$ transition with the absorption of k photons ( $\alpha$, p-discrete levels). For the resonance which corresponds to this transition, we calculate the following values:

$$
\begin{gathered}
\delta \omega(p \alpha \mid k)=\int{ }^{\prime} d \omega \operatorname{Im} E_{\alpha}(\omega)\left(\omega-\omega_{p \alpha} / k\right) / N, \\
\mu_{\mathrm{m}}=\int^{\prime} d \omega \operatorname{Im} E_{\alpha}(\omega)\left(\omega-\omega_{p \alpha} / k\right)^{\mathrm{m}} / N,
\end{gathered}
$$

where $\int$ ' $d \omega \operatorname{Im} E_{\alpha}$ is the normalizing multiplier; $\omega_{p \alpha}$ is position of the non-shifted line for atomic transition $\alpha-p, \delta \omega(p a l k)$ is the line shift under k-photon absorption and $\omega_{p \alpha}=\omega_{p \alpha}+k \cdot \delta \omega(p \alpha \mid k)$. The first moments $\mu_{1}, \mu_{2}$ and $\mu_{3}$ determine the atomic line center shift, its dispersion and coefficient of the asymmetry. To calculate $\mu_{m}$, we need to get an expansion of $E_{\alpha}$ to PT series: $E_{\alpha}=\sum E_{\alpha}{ }^{(2 k)}\left(\omega_{0}\right)$. To get this expansion, we use method, based on the Gell-Mann and Low adiabatic formula for $\delta E \alpha$, proposed in $[7,13]$. The representation of the $S$ - matrix in the form of PT series induces the expansion for $\delta E_{\alpha}$ :

$$
\begin{gathered}
\delta E_{\alpha}\left(\omega_{0}\right)=\lim _{\gamma \rightarrow 0} \gamma \sum_{k_{1} k_{2} \ldots k_{n}} a\left(k_{1}, k_{2}, \ldots, k_{n}\right), \\
I_{\gamma}\left(k_{1}, k_{2}, \ldots, k_{n}\right)=\prod_{j=1} S_{\gamma}^{(k)}, \\
S_{\gamma}^{(m)}=(-1)^{m} \int_{-\infty}^{0} d t_{1} \ldots \\
\ldots \int_{-\infty}^{t_{m}-1} d t_{m}\left\langle\Phi_{\alpha}\left|V_{1} V_{2} \ldots V_{m}\right| \Phi_{\alpha}\right\rangle, \\
V_{j}=\exp \left(1 H_{0} t_{j}\right) V\left(r t_{j}\right) \exp \left(-1 H_{0} t_{j}\right) \exp \left(\gamma t_{j}\right) .
\end{gathered}
$$

Here $H$ is the atomic hamiltonian, $a\left(k_{1}, k_{2}, \ldots, k_{n}\right)$ are the numerical coefficients. The structure of the matrix elements $S_{\gamma}^{(m)}$ is in details described in $[15,16]$. After sufficiently complicated one can get the expressions for the line moments. Let us present results for the Gaussian laser pulse $[15,20]$ :

$$
\begin{gathered}
\delta \omega(p \alpha \mid k)=\{\pi \Delta /(k+1) \mathrm{k}\}\left[E\left(p, \omega_{p \alpha} / k\right)-\right. \\
\left.-E\left(\alpha, \omega_{p \alpha} / k\right)\right], \\
\mu_{2}=\Delta^{2} / \mathrm{k} \\
\mu_{3}=\left\{4 \pi \Delta^{3} /[k(k+1)]\right\}\left[E\left(p, \omega_{p \alpha} / k\right)-\right. \\
\left.-E\left(\alpha, \omega_{p \alpha} / k\right)\right], \\
\text { where } E\left(j, \omega_{p \alpha} / k\right)= \\
=0,5 \sum_{p_{i}} V_{j p i} V_{p i j}\left[\frac{1}{\omega_{j p_{i}}+\omega_{p \alpha} / k}++\frac{1}{\omega_{j p_{i}}-\omega_{p \alpha} / k}\right]
\end{gathered}
$$

The summation in (8) is fulfilled on all states of atomic system. For the Lorentzian pulse the expressions are given in ref [15]. In a case of the laser pulse with shape $c h^{-1}[t / D]$ it is necessary to carry out a direct numerical calculation (we did it) or use different approximations to simplify the expressions. Indeed, the last procedure may result in a great mistake. Each term in equations (8) for $\delta \omega$ is formally similar to the known expression for off-resonant shift of atomic level ( $p$ or $\alpha$ ) in the monochromatic emission field with frequency $\omega_{p \alpha} / k$. However, here these values have other physical essence. When $k \rightarrow \infty$ (an infinite little laser pulse central frequency) the formula for $\delta E$ gives the correct expression for energy level shift in the stationary field. The expressions (8) for $\delta \omega$ and $\mu_{n}$ describe the main characteristics of the absorption line near resonant frequency $\omega_{p \alpha} / k$. One can see that these characteristics are determined not only by the radiation frequency, but also by the quantiness of the process. For example, the line shift is proportional $1 /(k+I)$, but no - to value of $1 / k$, as one can wait for. Under $k=1$ there is an additional non-standard term. Under availability of the additional DC electric field one must use a basis of the wave functions of atom, dressed by an external field (laser one, if this field is more intensive that an electric one; and other versa, if it is more weak one) in calculating the corresponding matrix elements. Note that this is an original moment of this paper. Another original moment is connected with using the consistent QED gauge invariant procedure for generating the atomic functions basises (optimized basises) $[13,14]$. It will be shown below that this approach allows getting the results in an excellent agreement with experiment and they are more precise in comparison with analogous data, obtained with using non-optimized basises. The details of the numerical procedure are presented in ref. [13-20].

\section{Results and discussion}

Let us present the results of calculating the multiphoton resonances spectra characteristics for atomic systems in a stochastic laser field and also DC electric and laser field and show the possibilities for sensing a structure of the stochastic, multi-mode laser pulse and photon-correlation effects for atomic (and nano-optical) systems in this field. At first we present the results of the numerical calculation for the three-photon resonant, four-photon ionization profile of atomic hydrogen ( $1 \mathrm{~s}-2 \mathrm{p}$ transition; wavelength $=365 \mathrm{~nm})$. In figure 1 we present the shift 


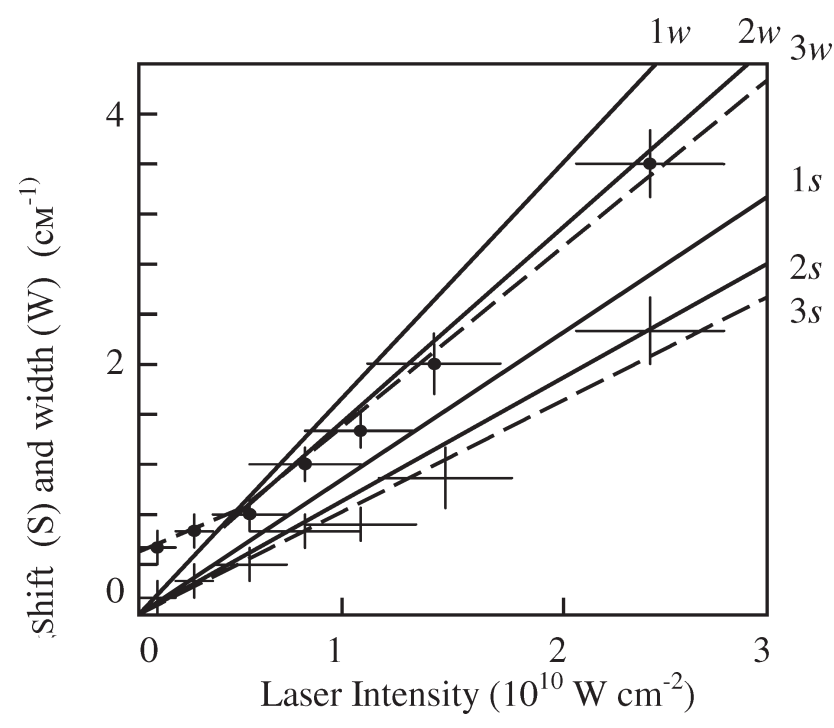

Fig.1. Shift (S) and width (W) of resonant profile as laser intensity function: experiment $-\mathrm{S}_{3}, \mathrm{~W}_{3}$ (Keller, Ligare, Brewer); theory of Zoller $-\mathrm{S}_{1}, \mathrm{~W}_{1}$ and our results- $\mathrm{S}_{2}, \mathrm{~W}_{2}$.

$\mathrm{S}(=\delta \omega)$ and width $\mathrm{W}$ of the resonance profile as the function of the mean laser intensity at the temporal and spatial center of the UV pulse: experimental data $\mathrm{S}_{3}, \mathrm{~W}_{3}$ (Kelleher, Ligare and Brewer [9]; multimode Gauss laser pulse with bandwidth $0.25 \mathrm{~cm}^{-1}$; full width at half of one), theoretical calculation results on the basis of the stochastic differential equations method $S_{1}$ and $W_{1}$ by Zoller [10]) and results of our calculation: $\mathrm{S}_{2}, \mathrm{~W}_{2}$. At first, one can see the excellent agreement between the theory and experiment. At second, a comparison of these results with analogous data for a Lorentzian laser pulse [15] shows that the corresponding resonance shift obtained with the gaussian pulse is larger the shift, obtained with Lorentzian pulse at $\sim 3$ times. This is an evidence of the photon-correlation effects and stochasticity of the laser pulse. Under availability a weal DC electric field this relationship is changed. Further let us consider the numerical calculation results for three-photon transition $6 \mathrm{~S}-6 \mathrm{~F}$ in the $\mathrm{Cs}$ atom (wavelength $1,059 \mu \mathrm{m}$ ). The detailed experimental study of the multi-photon processes in Cs atom has been carried out in ref. [11]. In this paper it is experimentally studied a statistics of the laser radiation and there are measured the characteristics of the multi-photon ionization. According to [11], the lines shift is linear to respect to the laser intensity (laser intensity is increased from 1,4 to 5,7 10(7) $\mathrm{W} / \mathrm{cm}^{2}$ ) and is equal (a case of the gaussian multimode laser pulse): $\delta \omega(p \alpha \mid k)=b I$ with $b=(5,6+-0,3)$ $\mathrm{cm}^{-1} / \mathrm{GW} \cdot \mathrm{cm}^{-2}$ ( $b$ is expressed in terms of energy of the three-photon transition $6 \mathrm{~S}-6 \mathrm{~F})$. The corresponding shift obtained with coherent (one-mode) laser pulse is defined as follows: $\delta \omega_{0}(p \alpha \mid k)=a I$, $a=2 \mathrm{~cm}^{-1} / \mathrm{GW} \cdot \mathrm{cm}^{-2}$. Theoretical values, received in ref. $[15,20]$ with using no-optimized atomic basises, are as follows: i). for soliton-like laser pulse: $\delta \omega(p \alpha \mid k)=b I, b=6,7 \mathrm{~cm}^{-1} / G W \cdot \mathrm{cm}^{-2}$; ii). for the gaussian multi-mode pulse (chaotic light): $\delta \omega(p \alpha \mid k)=b I$ with $b=5,8 \mathrm{~cm}^{-1} / \mathrm{GW} \cdot \mathrm{cm}^{-2} ;$ iii). for the coherent one-mode pulse: $\delta \omega_{0}(p \alpha \mid k)=a I$, $a=2,1 \mathrm{~cm}^{-1} / \mathrm{GW} \cdot \mathrm{cm}^{-2}$. The analogous theoretical values, received in our calculation within described above S-matrix formalism, are the following: i). the gaussian multi-mode pulse (chaotic light) $\delta \omega(p \alpha \mid k)$ $=b I, b=5,63 \mathrm{~cm}^{-1} / G W \cdot \mathrm{cm}^{-2}$; ii). the coherent onemode pulse: $\delta \omega_{0}(p \alpha \mid k)=a I, a=2,02 \mathrm{~cm}^{-1} / G W \cdot \mathrm{cm}^{-2}$; iii). the soliton-like laser pulse: $\delta \omega(p \alpha \mid k)=b I$, $b=6,5 \mathrm{~cm}^{-1} / G W \cdot \mathrm{cm}^{-2}$; One can see that for the with multi-mode pulse, the radiation line shift is significantly larger (in $\sim 3$ times), then the corresponding shift, which is obtained for single-mode pulse. One can see that the radiation line shift is enhanced by the photon-correlation effects. In fig. 2 we present

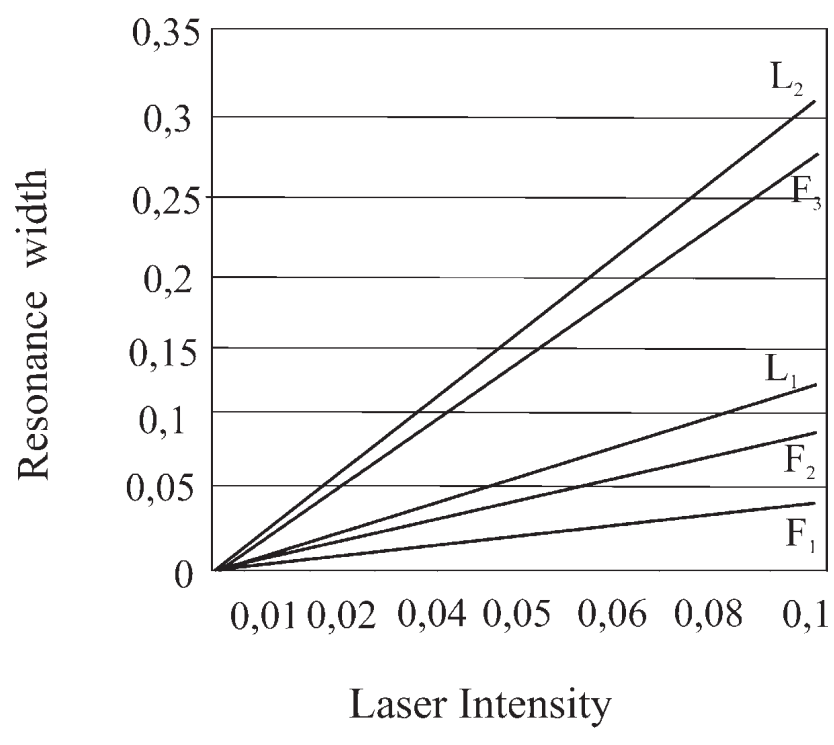

Fig.2. Resonance width (W) of resonant profile as laser intensity function (see notations in the text)

the results of calculating width for multi-photon resonance on transition $6 \mathrm{~S}-6 \mathrm{~F}$ in spectrum of the caesium Cs atom (wave-length $1.059 \mu \mathrm{M}$ ) in dependence upon an intensity of laser pulse. The following notations are used: L1, L2- multi-mode pulse with line widths $0.03 \mathrm{~cm}^{-1}$ and $0.15 \mathrm{~cm}^{-1}$ correspondingly (soliton-like laser pulse); F1- one-mode coherent Lorentzian pulse; F2 and F3- multi-mode gaussian pulse with line widths $0.03 \mathrm{~cm}^{-1}$ and $0.15 \mathrm{~cm}^{-1}$ corre- 
spondingly. One can see that photon-correlation effects enhance the radiation line shift and it is greater for stochastic light in comparison with coherent one. The degree of this enhancement may be considered as quantitative measure of the stochasticity of the multi-mode laser pulse and the photon-correlation effect contribution. An enhancement factor for situation in fig. 2 is equal 4.01 (for the gaussian pulse in comparison with coherent one) and 4,51 (for solitonlike pulse). In conclusion we consider a process of the multi-photon ionization for atomic system in the gaussian laser pulse field with frequency $\omega$ and DC electric field with the field strength $f$. We have carried out the calculation of the two-photon $\Gamma_{2}$ and three-photon $\Gamma_{3}$ ionization probability for the process: $N a+n \omega \rightarrow N a+e^{-}$by circularly-polarized light (magnetic field here is not taken into account). In fig. 3 we present a dependence of the value $\Gamma_{2} / \Gamma_{3}$ upon the parameter $Z_{0}=2 \omega /\left(2 f^{2 / 3}\right)$ : 1 -is corresponding to curve for $f=10^{7} \mathrm{~V} / \mathrm{cm}$; $2-f=5 \cdot 10^{7} \mathrm{~V} / \mathrm{cm}$; a- the results of the analytical approach $[21,22]$; b- our results. The detailed analysis shows that our results are more exact in comparison with data [21], obtained within simplified analytical model [19-22]. From the physical point of view, we have here an example of the nonmonotonous dependence for number of the absorbed photons upon parameters of the external laser and DC electric field. It is important to note that this dependence can be also considered as test for sensing a structure of the stochastic, multi-mode laser pulse field and photon-correlation features of atomic systems in a stochastic laser field and DC electric field.

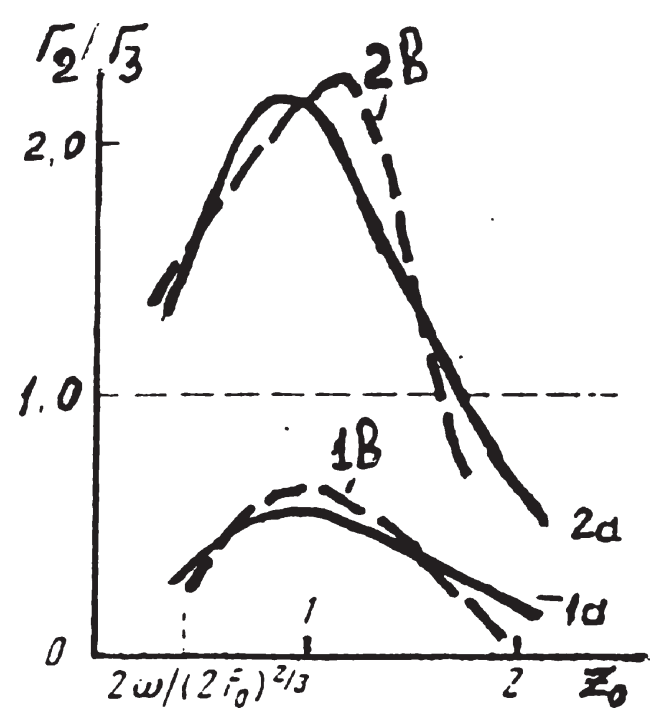

Fig.3. The dependence of the value $\Gamma_{2} / \Gamma_{3}$ upon the parameter $Z_{0}=2 \omega /\left(2 f^{2 / 3}\right)$ (see text)

\section{References}

1. Kleppner D., Chun-Ho I., Welch G.R., Irregular Atomic Systems and Quantum Chaos, Ed. J.C.Gay, N-Y.: Kluwer, 1990. - P.21-48.

2. Benvenuto F., Casati G., Shepelyansky D.L., Rydberg Stabilization of atoms in strong fields: "magic"mountain in chaotic sea// Z.Phys.B. 1994. - V.94. - P.481-486.

3. Delone N.B., Krynov V.P. Atom in a strong light field. - M.: Atomizdat, 1994.

4. Letokhov V.S. Non-linear selective photo processes in atoms and molecules. - M.: Nauka, 1993.

5. Plummer M., Noble C.J., Non-hermitiam dynamics of argon atoms at $248 \mathrm{~nm} / /$ J.Phys. B: At. Mol. Opt. Phys. - 2003. - Vol.36. - P.L219L226.

6. Staudt A., Keitel C.H., Stabilization of helium in intense high-frequaency laser pulses beyond the dipole approximation// J.Phys. B: At. Mol. Opt. Phys. — 2003. — Vol.36. - P.L203-L209.

7. Ivanov L.N., Ivanova E.P., Knight L. Energy Approach to consistent QED theory for calculation of electron-collision strengths//Phys.Rev.A. 1993. - Vol.48,N6. - P.4365-74

8. Ullrich C.A.,Erhard S.,Gross E.K.U. Density Functional Approach to Atoms in Strong Laser Pulses// Superintense Laser Atoms Physics. - NY.:Kluwer,1986. - P.1-18.

9. Kelleher D.E., Ligare M., Brewer L.R. Resonant four photon ionization of atomic hydrogen// Phys.Rev.A. - 1985. - Vol.31,N4. - P.27472751.

10.Zoller P. Stark Shifts and resonant multi-photon ionization in multimode laser fields// J.Phys.B: At. Mol. Opt. Phys. - 1982. — Vol.15,N8. - P.29112933.

11. Lompre L-A., Mainfrau G., Manus C., Marinier J.P., Laser Light statistics and band-width effects in resonant multi-photon ionization of caesium atoms1.059 mm // J.Phys. B: At. Mol. Opt. Phys. 1981. - Vol.14. - P.4307-4326.

12. Luc-Koenig E., Lyras A., Lecomte J. - M., Aymar M. Eigenchannel R-matrix study of two-photon processes in magnesium// J.Phys. B: At. Mol. Opt. Phys. - 1997. - Vol.30. - P.5213-5232.

13. Glushkov A.V.,Ivanov L.N. DC Strong-Field Stark-Effect: consistent quantum-mechanical approach// J.Phys. B: At. Mol. Opt. Phys. - 1993. — Vol.26,N16. - P.L379-L386.

14. Glushkov A.V., Ivanov L.N. Radiation Decay of Atomic States: atomic residue and qauge noninvariant contributions// Phys. Lett.A. - 1992. - Vol.170,N1. - P.33-37.

15. Glushkov A.V., Ambrosov S.V., Malinovskaya S.V. Spectroscopy of atom in laser field// Bound 
Volume of Paris-Meudon Observatory (Paris, France). - 1996. - P.148-152.

16. Glushkov A.V., Prepelitsa G.P., Polischuk V.P. etal QED Theory of nonlinear Interaction of the complex Atomic Systems with Laser field. Multiphoton Resonances// J. Techn. Phys. - 1997. Vol.38,N2. - P.219-224.

17. Glushkov A.V., Ambrosov S.V., Svinarenko A.A. etal, QED calculation of the super heavy elements ions: energy levels and hyperfine structure for different nuclear models// Nucl. Phys.A. - 2004. - Vol. 734. - P.E21-24.

18. Glushkov A.V.,Malinovskaya S.V., Svinarenko A.A., Cgarnyakova Yu.G., QED Calculation of Electron Satellites Spectra in Intense Laser Field in Multicharged Ion//Int.J.Quant.Chem. - 2004. Vol.99,N5. - P.673-678.

19. Svinarenko A.A. Multi-photon absorption in atoms and narrow-band semiconductors in crossed external fields //Photoelectronics. - 2003. - V.13. - P.137-142.

20. Glushkov A.V., Rusov V.D., Ambrosov S.V., Loboda A.V. Resonance states of compound super-heavy nucleus and EPPP in heavy nucleus collisions // In: New projects and new lines of research in nuclear physics, Eds. G.Fazio,F.Hanappe, Singapore :World Sci., 2003. - P.142-154.

21. Slonim V.Z., Dalidchik F.I., Impurity electroabsorption and photo destruction of negative ions in the presence of electric field // JETP. -1976. Vol.74,N6. - P.2057-2067.

22.Zhilich A.G., Monozon B.S., Multi-photon magnetic absorption in narrow-band semiconductors located in crossed fileds// JETP. 1980. - Vol.78,N. - P.1087-1098. 